\title{
Vuelo con destino al néctar de la fruta
}

\section{El investigador del Instituto de Neurociencias de la UMH Alejandro Gómez Marín descubre que la ley de potencias en el trazado de los garabatos humanos está presente en las trayectorias de las larvas de la mosca de la fruta}

\section{PATRICIA SANTOS}

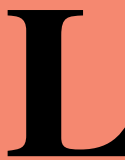

os plátanos, las peras o las naranjas sirven de alimento para la Drosophila melanogaster, conocida como la mosca de la fruta. Esta minúscula especie díptera posee unos sensores olfativos muy desarrollados, sus antenas le permiten orientarse hacia el alimento y con sus patas saborean el mundo. Cuando una mosca hembra adulta encuentra la fruta, deposita sus huevos para que puedan comer y desarrollarse. Además, la Drosophila melanogaster realiza ciertos comportamientos o conductas equiparables a las de los seres humanos. Puede escalar porque percibe la gravedad o siente miedo ante lo desconocido.

El investigador del Instituto de Neurociencias (IN), centro mixto de la Universidad Miguel Hernández (UMH) de Elche y el Consejo Superior de Investigaciones Científicas (CSIC), Alejandro Gómez Marín ha liderado un estudio titulado 'La velocidad y curvatura de la ley de potencia en la locomoción larval de Drosophila', junto a los investigadores de la Università degli Studi $\triangleright$

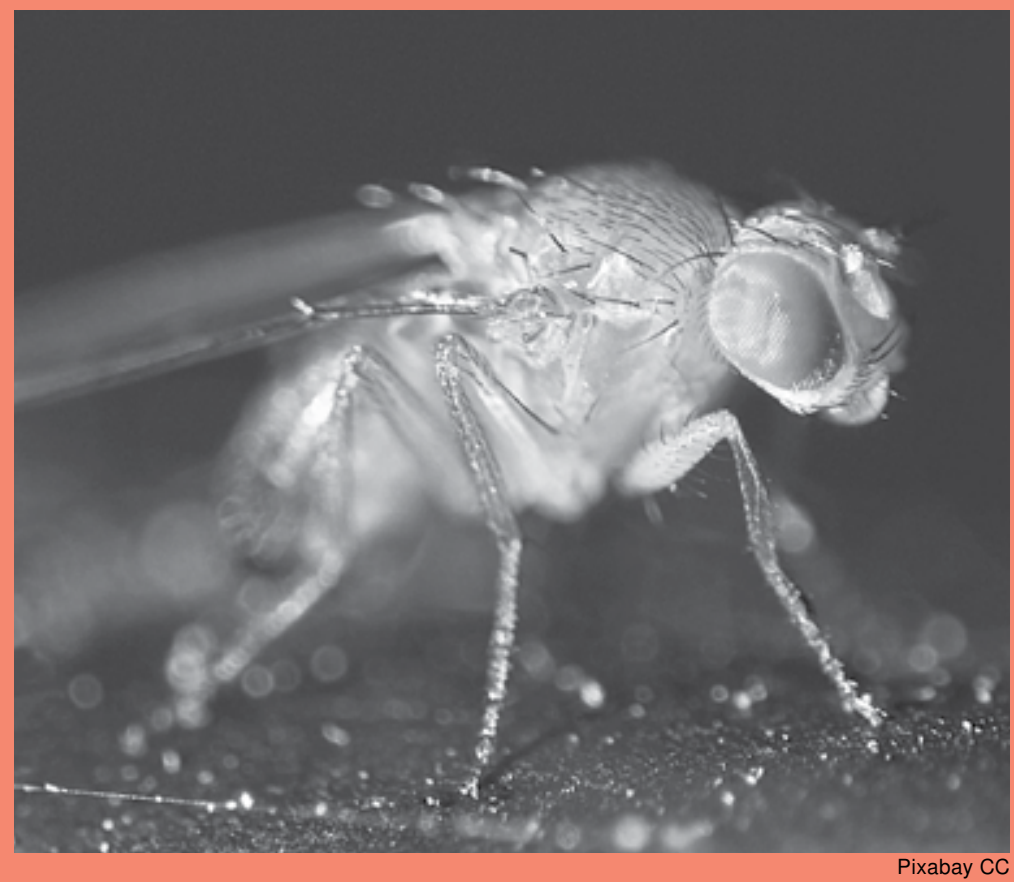


di Roma Tor Vergata y la Fondazione Santa Lucia de Italia Francesco Lacquaniti y Myrka Zago. En él se revela cómo la ley de potencias que relaciona la curvatura y la velocidad en el trazado de los garabatos humanos, también está presente en las trayectorias de las larvas de la mosca de la fruta, mientras buscan alimento.

Alejandro Gómez Marín explica que la idea del proyecto surgió a raíz de la organización de un curso sobre homologías de conductas, en el que se trataban cuestiones sobre las semejanzas y las diferencias del comportamiento entre especies. Gómez Marín conoció entonces a Francesco Lacquaniti, uno de los autores del estudio. Lacquaniti presentó su trabajo sobre la ley de potencias en humanos, ya que fue él quién la descubrió en los años 80. El investigador del IN aclara que ya a finales del siglo XIX, Binet y Courtier evidenciaron que cuando una persona dibuja un garabato en un papel, la velocidad con la que puede trazar el diseño es diferente en las curvas que en las líneas rectas. En el primer caso, disminuye; en el segundo, aumenta. "Lacquaniti y sus colaboradores descubrieron hace 35 años que tal dependencia sigue una ley matemática muy concreta e inesperada. Por tanto, el trazado covaría con la velocidad con la que se puede realizar el dibujo y se produce una restricción biológica fuerte entre velocidad y curvatura. Ahora acabamos de descubrir que esta invariancia conductual no sólo se da en humanos, sino también en insectos", concreta Gómez Marín. Este proceso es similar también cuando una persona conduce un coche. En rectas puede acelerar y adelantar, pero en las curvas reduce la velocidad.

Para explicar el fenómeno de la ley de potencias en humanos existen dos hipótesis generales. Una de ellas enfatiza que la ley es obra del córtex cerebral al realizar un plan justo antes de ejecutar el dibujo. La otra versa sobre la interacción en tiempo real de los músculos con el medio, sin necesidad de computaciones neuronales complejas.

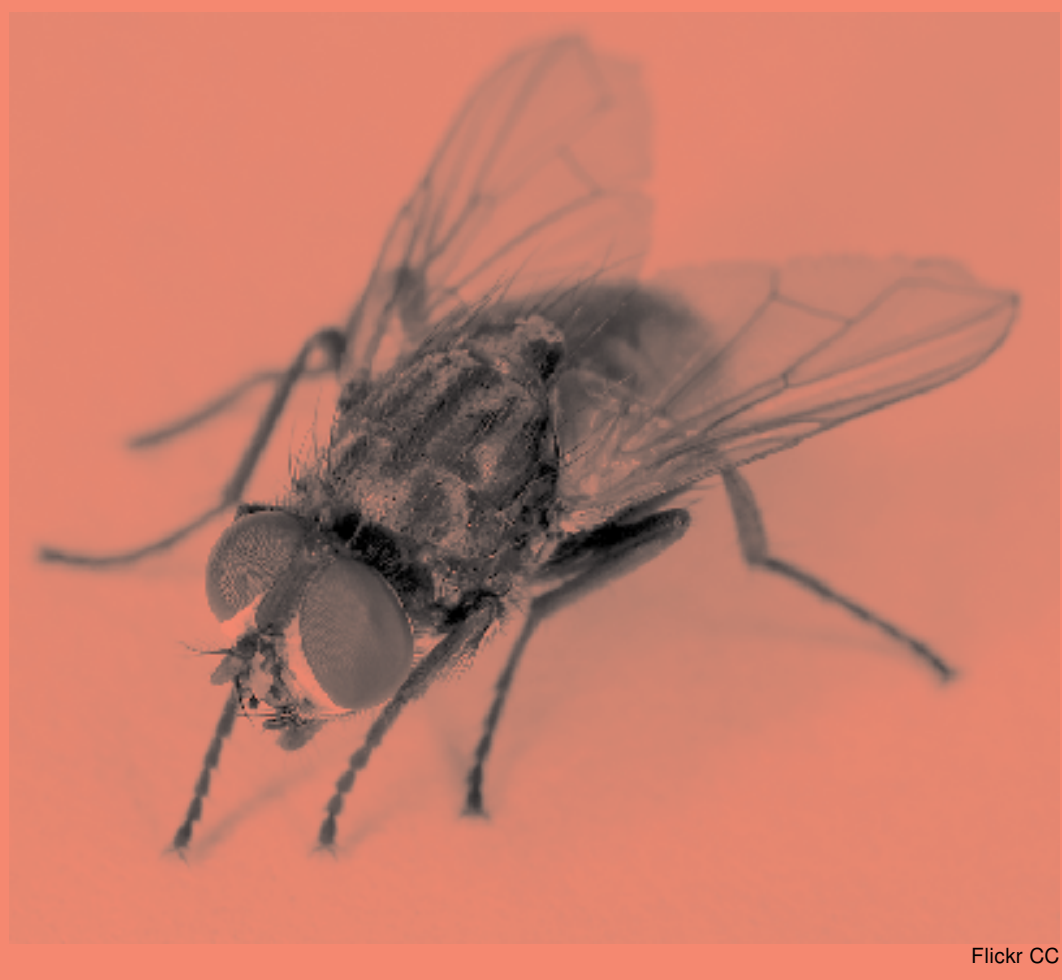

Flickr CC

\section{La Drosophila melanogaster realiza ciertos comportamientos o conductas equiparables a las de los humanos}

El investigador del Instituto de Neurociencias de la UMH analizaba trayectorias parecidas a un garabato humano, pero hecho por moscas. Se acordó del trabajo del Lacquaniti y probó por primera vez la ley de potencias del trazado de los garabatos humanos en las trayectorias que dejan las larvas de Drosophila melanogaster. "El recorrido que realizan las moscas para buscar comida o navegar en un gradiente de olor, una de sus mayores ocupaciones en su corta vida, se asemejaba a los trazados que una persona efectúa al dibujar. Si se dijera que esas trayectorias las ha realizado un humano, en vez de una larva, parecería verdad. La diferencia radica en que las personas sí poseen córtex cerebral y unas $86.000 \mathrm{mi}$ llones de neuronas (contando una por segundo tardaríamos 3.000 años en contarlas todas); mientras que las moscas de la fruta no tienen córtex y su capacidad neuronal, aunque destacable, es mucho menor: las larvas de la mosca de la fruta sólo poseen unas 10.000 neuronas en su minúsculo cerebro", puntualiza Gómez Marín.

El estudio realizado por Alejandro Gómez Marín, junto con los investigadores Fran- cesco Lacquaniti y Myrka Zago, sobre la ley de potencias en la Drosophila sugiere que la mosca de la fruta efectúa un comportamiento modelado por la selección natural. “La Drosophila no sabe de matemáticas ni de leyes, pero lleva a cabo conductas cuyos patrones reflejan cierta eficiencia en su movimiento", aclara el científico del IN. Los investigadores utilizaron tres grupos de larvas que expusieron a diferentes condiciones para llevar a cabo los experimentos. La alternativa de aproximación o approach consistía en colocar una fuente de olor en el lado opuesto al de las larvas. La condición pasar de largo u overshoot versaba sobre el recorrido que hacía la Drosophila cuando olía la fruta que estaba suspendida en el aire. El científico del IN expresa: "En overshoot, el trayecto que realizaba la larva al reptar, se asemejaba a los garabatos espontáneos hechos por niños o adultos humanos. La Drosophila sí podía percibir el aroma de la comida, pero pasaba de largo y volvía a dar la vuelta". En la alternativa de dispersión o dispersal la larva buscaba el alimento, aunque los investigadores no se lo pusieran, ya que su principal objetivo consiste en comer y crecer.

El científico del IN manifiesta que investigar con Drosophila melanogaster tiene muchas ventajas, ya que la mosca de la fruta tar- 


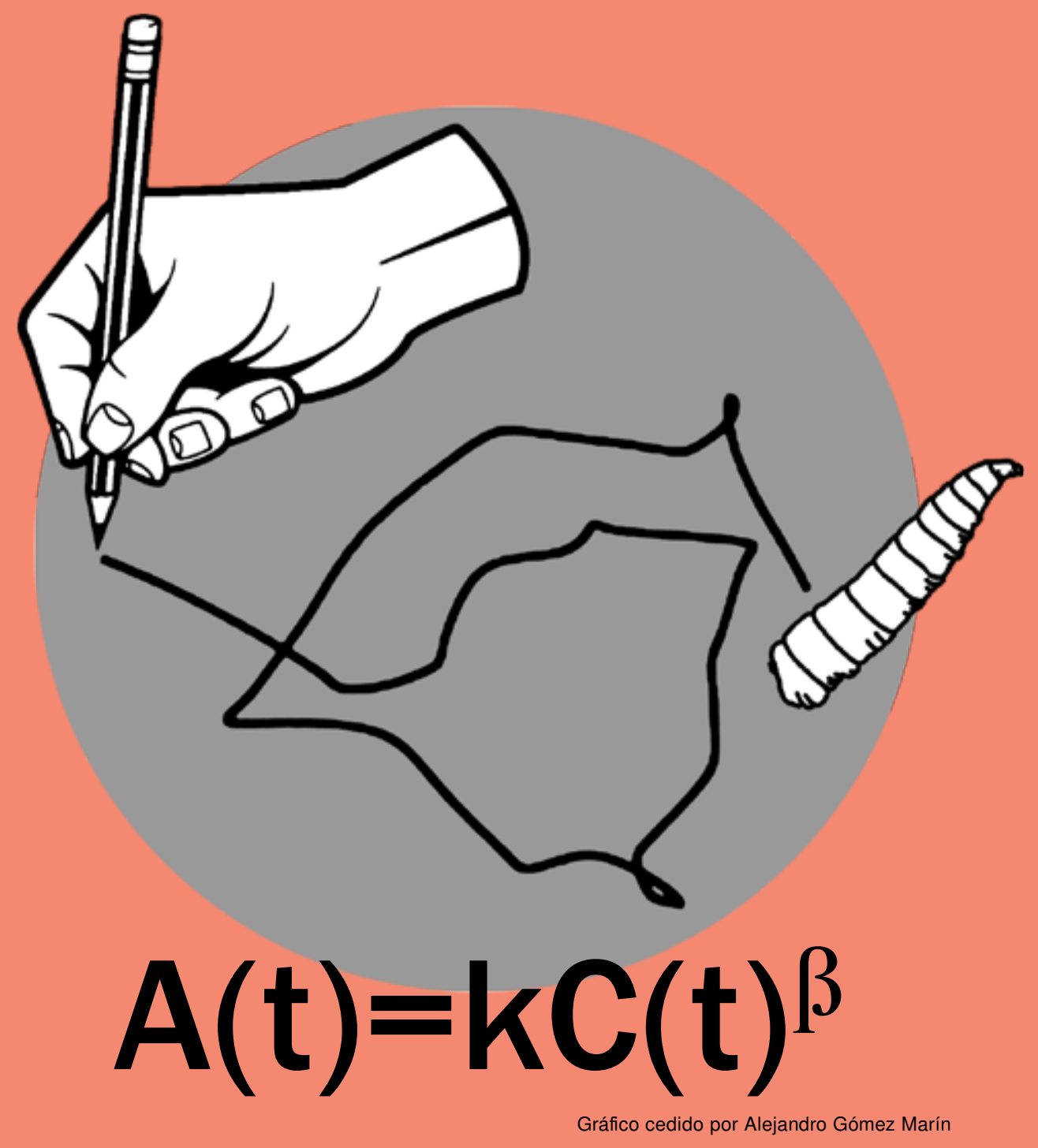

da dos semanas en convertirse en adulta: "Empieza siendo un huevo y se transforma en una larva que repta y se arrastra por la superficie. En 4 o 5 días triplica su tamaño. En la fase de pupa realiza una metamorfosis como las mariposas. Por último, llega la mosca adulta, cuya duración de vida ronda los dos meses". Asimismo, los investigadores descubrieron que el exponente de la fórmula matemática de la ley de potencias en la trayectoria de las larvas era $3 / 4$, mayor que el de los humanos al dibujar en papel, $2 / 3$. Recientemente, Francesco Lacquaniti ha realizado una serie de experimentos con humanos que consistía en dibujar elipses con la mano metida en un tanque de agua. Bajo estas condiciones, el exponente de la ley de potencias se acercaba a los $3 / 4$ de las larvas de Drosophila melanogaster. Alejandro Gómez Marín expresa que los experimentos realizados en la mosca de la fruta y en humanos indican la importancia no sólo de la geometría, sino de la dinámica, ya que el medio físico condiciona el exponente.

Por otra parte, el grupo de Comportamiento de los Organismos del IN, Behavior of Organisms Laboratory, encabezado por Gómez Marín, ha creado una aplicación que permite comprobar la ley de potencias en humanos al dibujar trayectorias a través de un ordenador, móvil o tablet. Durante unos segundos, las personas pueden hacer libremente garabatos, trazar curvas tales como una elipse o seguir un puntero. Transcurrido ese tiempo se muestra la velocidad y la curvatura, la contraposición de los logaritmos de velocidad angular y curvatura y el exponente conseguido. Las personas pueden comprobar si han alcanzado los 2/3. Además, el Grupo está construyendo un brazo robótico con el objetivo de comprobar si la ley de potencias que surge en sistemas biológicos también aparece en sistemas mecánicos. Alejandro Gómez Marín expresa que en ciencia todo puede ser interesante, ya que cuando un resultado no sale como en un principio se espera, mejor aún porque debes explicar y repensar todo el proceso: "Hemos tenido la suerte de abrir un camino para que los científicos puedan plantearse cosas que quizá ni se imaginaban sobre la ley de potencias. Por alguna razón, las larvas de Drosophila, y no sólo los humanos, realizan ese comportamiento. Será muy interesante comprobarlo en moscas modificadas genéticamente para así poder dilucidar cuantitativamente la contribución de grupos específicos de neuronas $y$, sobre todo, testarlo en otras especies animales tales como peces o ratones para establecer principios generales de comportamiento animal que abarquen desde el humilde gusano al curioso humano". 